\title{
sciendo
}

\section{Logical Form, Truth Conditions, and Adequate Formalization}

\author{
Mario Gómez-Torrente \\ National Autonomous University of Mexico (UNAM)
}

BIBLID [0873-626X (2020) 58; pp.209-222]

\begin{abstract}
I discuss Andrea Iacona's idea that logical form mirrors truth conditions, and that logical form, and thus truth conditions, are in turn represented by means of adequate formalization. I criticize this idea, noting that the notion of adequate formalization is highly indefinite, while the pretheoretic idea of logical form is often much more definite. I also criticize Iacona's claim that certain distinct sentences, with the same truth conditions and differing only by co-referential names, must be formalized by the same formula (in the same context). I criticize this claim, noting that it imposes implausible demands on adequate formalization. Finally, I offer some brief remarks on the connection between Iacona's ideas and the distinction between logical and non-logical constants.
\end{abstract}

\section{Keywords}

Co-referential names, formalization, logical constants, logical form, truth conditions.

In his book Logical Form (Iacona 2018), Andrea Iacona argues that the expression 'logical form' has been used with at least two different ideas in mind. One of the ideas, of old pedigree, is that a sentence has a peculiar kind of associated structure (its "logical form") that is crucially relevant (in ways that differ from theory of logic to theory of logic) to questions about what the sentence's logical properties areto whether it's logically valid, to questions about what it logically implies and what logically implies it, etc. The other, more recent idea, is that a sentence has an associated representation (its "logical form") which displays the compositional structure of the sentence at the syntactic and lexical levels. Iacona argues that the corresponding two ideas behind uses of 'logical form' are unlikely to be satisfiable by one single conception of logical form. 
Iacona then focuses on "logical form" in the sense of the first idea, logical form as a structure on which the logical properties of a sentence depend. He claims and argues that logical form in this sense cannot be something that depends entirely on intrinsic properties of a sentence (with this he is thinking, somewhat misleadingly, of certain (not really intrinsic) properties of the sentence, such as its graphical representation, its underlying syntax, and its lexical (as opposed to referential) meaning ${ }^{1}$ ). The claim about intrinsicality as normally understood is already evident from the fact that homonymous words appearing in the same sentence must, on any view, receive different representations in logical form. But Iacona argues at length for the claim (on his understanding of intrinsicality) in what I think is a potentially more controversial way, by focusing on indexicals and claiming (for example) that two occurrences of the same indexical in the same sentence (or argument) may have different referents and that in these cases it is just unavoidable to represent those occurrences differently in a logical formalization. Claims of this kind lead him to what I think is the main idea of the book, namely that the logical form (always in the "logical" sense of 'logical form') of a sentence (or better, of a sentence occurrence) must be a representation that mirrors closely the sentence's truth condition, understood as the proposition expressed by the sentence. Iacona then gives as a criterion for obtaining such a representation the idea that the logical form of a sentence (occurrence) ought to be given by an adequate formalization of it. The demand for a representation of truth conditions and its accompanying criterion appear, at least at first sight, to respect Iacona's claims about indexicals. If in This is the same as this the first occurrence of 'this' has a referent different from that of the second occurrence, then the truth condition of the sentence (occurrence) is certainly not appropriately represented by (say) the firstorder sentence $a=a$, and it seems adequate, at least in some contexts, to formalize the sentence by $a=b$, which does intuitively represent the proposition expressed by the sentence, at least according to the widely accepted direct reference view of indexicals.

\footnotetext{
${ }^{1}$ See p. 37, where the intrinsic properties of a sentence (occurrence) are characterized essentially as its context-independent properties, the "invariant properties that [a sentence] s possesses independently of how it is used in this or that context".
} 
Now, while I sympathize with Iacona's insistence that logical form is, if anything, something extrinsically related to a sentence (in the usual sense of extrinsicality) and that adequate formalization may in some contexts consist in representations that mirror closely the truth conditions of sentences, I think his ways of understanding the general relationship between logical form, adequate formalization, and truth conditions are not always well-taken. This is manifested in particular in some strong claims of Iacona's regarding the connection between logical form and adequate formalization. In what follows I will explain what the claims are and why I think they are problematic.

\section{Iacona on different, equally adequate representations of the same truth conditions}

Iacona's idea that truth conditions, and hence logical form, are represented by means of adequate formalizations seems to me somewhat crude and thus problematic. The basic reason, as we will see, is that the idea of adequate formalization is highly indefinite, while the pretheoretic idea of logical form (once we have settled on the "logical" notion of logical form) is often much more definite. ${ }^{2}$

One way to see this is through Iacona's claim that there are substantively different equally adequate formalizations of the same sentence, as long as they do not differ in truth conditions as he understands these. One example arises in the voice of an imaginary objector considered by Iacona:

consider the following sentences:

(21) Not all Martians are green

(22) Some Martians are not green

\footnotetext{
${ }^{2}$ In speaking of the "pre-theoretic" idea of logical form I ought perhaps note that I'm not thinking of an intuitive idea existing prior to any theoretical work in logic - there is obviously no such thing. I'm thinking of the idea or ideas of logical form implicit in the theoretical work of logicians and prior to theoretical (or meta-theoretical) work by philosophers of logic on the notion of logical form itself. Implicit ideas about logical form can be found in the work of logicians since Aristotle and his commentators, but explicit theoretical reflection on the notion begins at the earliest in the Middle Ages, and can only be said to have matured in recent times.
} 
The equivalence between (21) and (22) seems to hold for a purely logical reason, that is, the interdefinability of quantifiers. However, if logical form is determined by truth conditions, then [(21) and (22)] must be represented by the same formula. (Iacona 2018: 64)

The objector is then unhappy with this because it appears to make the equivalence between (21) and (22) trivial. Iacona grants to the objector that (21) and (22) have the same truth conditions, but argues that this is not a problem for his view, as the equivalence between (21) and (22) is not trivial because it is not trivial that they can be represented by the same formula. They can be both represented by ' $\sim \forall \mathrm{x}(\mathrm{Fx} \supset \mathrm{Gx})$ ', say, but it's not trivial that they can both be so represented.

However, what I find most troubling with the situation pointed out by the objector is not an epistemic difficulty about triviality of equivalence or triviality of the possibility of representation, but the simple fact that (21) and (22) do not appear to have the same logical form, on any (sensible) pre-theoretic understanding of (the logical notion of ) 'logical form'. Regardless of the indeterminacies and vagaries concerning the pre-theoretic idea of logical form, this, it seems to me, is sufficiently definite to exclude the possibility that (22) has as its logical form a representation involving a universal quantifier, period. However, if Iacona grants that (21) and (22) have the same truth conditions, and also that ' $\sim \forall \mathrm{x}(\mathrm{Fx} \supset \mathrm{Gx})$ ' adequately formalizes (22), then, since so does ' $\exists x(F x \wedge \sim G x)$ ', this implies the pre-theoretically absurd consequence that ' $\sim \forall \mathrm{x}(\mathrm{Fx} \supset \mathrm{Gx})$ ' and ' $\exists \mathrm{x}(\mathrm{Fx} \wedge \sim \mathrm{Gx})$ ' have the same logical form.

To me this suggests that Iacona's close linking of logical form with the (possibility of) adequate formalization is misguided. Adequate formalization is arguably a matter highly sensitive to the theoretical or practical context, while logical form, at least in some pre-theoretically central or paradigmatic cases, doesn't seem to be sensitive to context in that way. This emerges with some force from another part of Iacona's response to the objector above. According to Iacona, his view is not that distinct sentences with the same truth conditions must be represented by the same formula, but that distinct sentences with the same truth conditions can be represented by the same formula (and that this is not trivial in particular cases) (cf. 
Iacona 2018: 64). This possibility of representation must apparently be understood deontically, for Iacona argues that it is clearly permissible for a logic student, regardless of the context, to formalize (22) by ' $\forall \mathrm{x}(\mathrm{Fx} \supset \mathrm{Gx})$ ':

Imagine a written logic exam in which students are asked to formalize an argument containing [(21) and (22)], and suppose that one of them uses the formula [' $\forall \mathrm{x}(\mathrm{Fx} \supset \mathrm{Gx})$ '] to represent both sentences. In this case, it would be unfair for the teacher to mark the formalization as mistaken. (...) After all, the student might say, why should this difference matter, if it doesn't matter to the validity or invalidity of the argument? (Iacona 2018: 64-5)

To me, however, it seems evident that whether the teacher will (fairly) mark the formalization as mistaken is not an absolute matter, but something relative to the pedagogical context. It is certainly true that in some contexts it will be appropriate to formalize (22) by ' $\forall \forall \mathrm{x}(\mathrm{Fx} \supset \mathrm{Gx})$ '. But if part of the exercise is to show that (22) can be deduced from (21) via a proof in a calculus with its corresponding different rules for the universal and the existential quantifier (a perfectly natural exercise), then Iacona's student will appropriately get her response marked as mistaken. By contrast, the question whether (21) and (22) (or ' $\sim \forall \mathrm{x}(\mathrm{Fx} \supset \mathrm{Gx})$ ' and ' $\exists \mathrm{x}(\mathrm{Fx} \wedge \sim \mathrm{Gx})$ ') have the same logical form has, on any sensible pre-theoretic view, an absolute response: they don't.

Iacona has been misled by the frequent possibility of formalizing without guilt the distinct sentences (21) and (22) by the same formula into thinking that they must have the same logical form and hence, the same truth conditions. The right view of the situation may, nevertheless, be compatible with the idea that logical form mirrors truth conditions. Unlike Iacona, a defender of the view must reject the claim that (21) and (22) have the same truth conditions (a perfectly natural rejection under assumptions dear to Iacona, such as the assumptions typical of views of propositions as ordered structures of individuals, properties and other items). After all, (21) and (22) appear to talk of different quantificational properties. If (21) and (22) have different truth conditions, then they have different logical forms on Iacona's view. And this is compatible with the possibility of formalizing them by the same formula in some contexts, depending 
on the theoretical or practical needs at stake in those contexts.

Another way to see that the notion of adequate formalization is less definite than the notion of (representation of) logical form is via another objection considered by Iacona. The objector says:

Consider the following sentences:

(23) Donald is a drake

(24) Donald is a male duck

Since (23) and (24) are synonymous, they have the same truth conditions. Therefore, on the assumption that formulas represent truth conditions, (23) is adequately formalized as $F a \wedge G a$. This entails that the following argument is formally valid:

[E] (23) Donald is a drake / (25) Donald is a duck

That is, [E] turns out to have the following form:

[F] (26) $F a \wedge G a /(27) G a$

So it seems that the consequence we get is that materially valid arguments, which should be counted as formally invalid, turn out trivially formally valid. (Iacona 2018: 65)

To this Iacona reasonably replies that accepting that synonymy gives rise to some formal validities doesn't imply that all materially valid arguments are formally valid. One may draw the line somewhere, accepting that synonymy gives rise to formal validity but other forms of material or necessary equivalence don't; and Iacona does say that a form of equivalence that gives rise to formal validity is "conceptual analysis" (cf. Iacona 2018: 66).

However, one must be aware that the ideas that logical form represents truth conditions and that adequate formalization represents truth conditions and hence codifies logical form are too indeterminate to support unequivocally any way of drawing the line. Should we or should we not say that certain metaphysically necessarily equivalent sentences have the same truth conditions and hence (on Iacona's view) the same logical form? If someone says that the argument

[A] The liquid in John's glass is water / The liquid in John's glass is $\mathrm{H}_{2} \mathrm{O}$ 
is valid because its premise and its conclusion have the same truth conditions (assuming, with much of the philosophical literature, that necessarily water is $\mathrm{H}_{2} \mathrm{O}$ ), and hence that an adequate formalization of it is

[B] The $F$ is $G$ / The F is G,

on what grounds can we say that this person is mistaken? I do think she is mistaken, because I think that the premise and conclusion in [A] have different logical forms, period, and hence that [B] doesn't adequately represent those logical forms. But the point is that it's hard to see how Iacona could justify non-arbitrarily this claim, because his view of logical form is too indeterminate to imply this. For it seems that it can perfectly well be held that premise and conclusion in [A] do have the same truth conditions and $[\mathrm{B}]$ is an adequate formalization of $[\mathrm{A}]$ (especially given that it's not difficult to imagine contexts where the formalization of [A] by [B] would be unimpeachable), and that they do not have the same truth conditions (they involve mention of different properties, for example) and [B] is not an adequate formalization of [A]. (In fact, as we will see below, elsewhere Iacona goes beyond his claim about "conceptual analysis" and embraces the view that the closely related argument

Hesperus is a star / Phosphorus is a star

is unequivocally formally valid, despite the fact that this would appear to be an entirely arbitrary choice given his framework.) Thus it seems to me that the flexibility and indefiniteness of the notion of adequate formalization in this case again indicates that Iacona's close linking of logical form with the (possibility of) adequate formalization is misguided.

\section{Iacona on identity of reference and formalization}

A worrisome problem for Iacona arises in a type of situation where, apparently inconsistently with his claim above that distinct sentences with the same truth conditions need not be formalized by the same formula, he repeatedly claims that certain distinct sentences with the same truth conditions must be formalized by the same formula (at least in the same context). This latter claim is more consistent 
with the root view that logical form must mirror truth conditions, but it is more worrisome because it imposes harder demands on adequate formalization.

According to Iacona, a rule of formalization that follows from his conception of logical form is one that

prescribes that distinct names denote distinct objects:

(N2) If $n_{1}$ and $n_{2}$ are logically distinct, $n_{1}$ denotes $x$, and $n_{2}$ denotes $y$, then $x \neq y$.

Contrapositively, if $x=y$, then it is not the case that $n_{1}$ and $n_{2}$ are logically distinct, $n_{1}$ denotes $x$, and $n_{2}$ denotes $y$. (Iacona 2018: 71)

The notion of logical distinctness in (N2) is defined in terms of the notion of adequate formalization:

Definition 6.2.2 Two occurrences of names $n_{1}$ and $n_{2}$ are logically distinct if and only if they are represented by distinct individual constants in an adequate formalization of the sentences in which they occur. (Iacona 2018: 70)

(N2) thus implies that when two names are represented by different individual constants in an adequate formalization, the objects they denote must be distinct; or, in other words, that the same object can only be represented (in the same context of formalization) by one and the same individual constant. "Distinct names must denote distinct objects" (Iacona 2018: 70).

An imagined objector questions this consequence of Iacona's view as follows:

According to (N2), both (34) and (35) are adequately formalized as Fa:

(34) Hesperus is a star

(35) Phosphorus is a star

Consequently, we get that the following arguments are both formally valid:

[Q] (34) Hesperus is a star / (34) Hesperus is a star

[R] (34) Hesperus is a star / (35) Phosphorus is a star

This is an unwelcome result. [Q] and [R] are both valid, in the sense that they are necessarily truth-preserving. But only [Q] should be classified as formally valid, because only the validity of [Q] depends on purely structural properties of its premise and conclusion. (Iacona 2018: 78) 
To this Iacona responds that "contrary to what is usually believed, it is questionable that $[\mathrm{R}]$ should not be classified as formally valid" (ibid.), adding:

Of course, $[\mathrm{R}]$ significantly differs from [Q], in that in order to recognize that $[R]$ is formally valid we need to know that 'Hesperus' and 'Phosphorus' refer to the same planet. However, as in the case of 'Aristotle' and 'the Stagirite', the crucial question is whether such difference matters to formalization, and it is reasonable to say that it does not. (Iacona 2018: 79)

Iacona supports this reply by giving examples of cases where an analogous difference does not appear to matter to formalization, one of which is the mentioned case of 'Aristotle' and 'the Stagirite':

Suppose that you read the following passage in an old-fashioned book on ancient philosophy: "If Aristotle spent twenty years in the Academy and studied extensively the Dialogues, he admired Plato. But we know that he spent twenty years in the Academy and studied extensively the Dialogues. Therefore, the Stagirite admired Plato". The author of the book seems to reason validly. The following argument, which provides a simplified statement of the author's reasoning, seems valid:

[G] (17) If Aristotle spent etc., then Aristotle admired Plato (18) Aristotle spent etc.

(16) The Stagirite admired Plato

The apparent validity of [G] is easily explained on the assumption that ['Aristotle admired Plato'] and (16) are adequately formalized as Rab:

[H] (19) $T a \wedge \mathrm{Sad} \supset \mathrm{Rab}$

(20) $\mathrm{Ta} \wedge \mathrm{Sad}$

(21) $R a b$

This formalization of [G] accords with (N2). Since 'Aristotle' and 'the Stagirite' denote the same individual, (N2) entails that they are logically identical. (Iacona 2018: 74-5)

I find Iacona's reply objectionable, and again not just because I think that it is simply a pre-theoretically evident fact that Hesperus is a star and Phosphorus is a star have different logical forms (which I do), but for reasons which should worry Iacona given other things he accepts. 
It seems clear again that Iacona is relying for his reply on the flexibility and context-dependency of the notion of adequate formalization, while this flexibility and context-dependency might be used to argue for the exactly opposite claims, making Iacona's preferred view arbitrary. It is clear that in the 'Aristotle' and 'the Stagirite' example what makes it appropriate to formalize both names by the same constant is the fact that the author of the book and the people to whom it is addressed (and the readers of Iacona's book) know full well that 'Aristotle' and 'the Stagirite' denote the same ancient philosopher. But think by contrast of a book about theoretical arithmetic written in 1987, just after it became known that Euler's sum of powers conjecture has counterexamples for the power 4 but before it was known that the sum of powers of 4 that provides the smallest counterexample is $422,481^{4}$, also known as $31,858,749,840,007,94$ $5,920,321 .^{3}$ The author of the book could perfectly well introduce a constant $\underline{\underline{e}}$ to designate this smallest counterexample, whose identity was unknown, and still of course use ' $31,858,749,840,007,9$ $45,920,321$ ' as a name for the same number in some other part of the same theoretical context in the book. (I'm assuming, like Iacona with 'the Stagirite', and in agreement with much of the literature on decimal Arabic numerals, that these are essentially names. ${ }^{4}$ ) It's clear that no one can fault the author of the book for formalizing inadequately the sentences in which reference to this number is made; her formalization of the number by means of both $\underline{e}$ and ' $31,858,749$ ,840,007,945,920,321' is perfectly acceptable. Furthermore, if she

${ }^{3}$ Euler's falsified conjecture said that for all natural numbers $n$ and $k$ greater than 1 , if the sum of $n k$ th powers of natural numbers is itself a $k$ th power, then $n \geq k$. This was falsified in the 1960's for $k=5$. In 1986, Noam Elkies found counterexamples for $k=4$. Then in 1988 Roger Frye showed that $422,481^{4}=95,800^{4}+$ $217,519^{4}+414,560^{4}$ provides the smallest counterexample.

${ }^{4}$ See e.g. Abbott 2010, where the idea is repeatedly emphasized that "some nouns that are not spelled with a capital letter nevertheless behave syntactically and semantically like proper names: words for numbers - like eight or ninetyare prominent examples" (p. 4); elsewhere (p. 49) she makes it clear that "words for numbers" include decimal Arabic numerals. Many authors in the tradition of the "new theory of reference" specifically view Arabic numerals as semantically non-descriptive singular terms (see e.g. Richard 1986, Soames 1987, 50; GómezTorrente 2019, ch. 4). 
had reasoned without further ado that "since $\underline{e}$ is a counterexample to Euler's conjecture, so is $31,858,749,840,007,945,920,321$ ", she would intuitively not be reasoning validly, ${ }^{5}$ and her book would have been rejected (or sent back to her accompanied by stern complaints of the referees).

Contrary to what Iacona says in his reply to the imagined objector, a difference in co-referential names may matter to formalization. Distinct names in an intuitively adequate formalization need not denote distinct objects. This dissolves once more the close link that Iacona sees between adequate formalization and the representation of truth conditions, for the representation of (identity of) truth conditions need not go hand in hand with adequate formalization. The idea of adequate formalization is again shown to be less definite than the idea of (representation of) truth conditions, and less definite than the idea of logical form. Again we see no convincing argument for the thesis that (representation of) truth conditions must yield (an adequate representation of) logical form, given the close link of truth conditions with adequate formalization. There is no such close link, which is compatible with the pre-theoreticallly clear view that $\underline{e}$ is a counterexample to Euler's conjecture and 31,858,749,840,007,945,920,321 is a counterexample to Euler's conjecture differ in logical form (just as Hesperus is a star and Phosphorus is a star differ pre-theoretically in logical form).

\section{The distinction between logical and non-logical expressions and some final remarks}

In some brief remarks toward the end of the book, Iacona says that his view of logical form as given by a representation of truth condi-

\footnotetext{
${ }^{5}$ I take the claim that the reasoning would be intuitively invalid (and the claim about the book referees' reaction) as obvious, though of course Iacona could suggest (and a referee of the present paper has suggested) that the reasoning might count as valid in some deeper theoretical sense, unrelated to the way logical validity is actually evaluated by mathematicians when simple first-order arithmetical reasonings are at stake. Even if we were to concede this, the substantive point in this section would stand, namely that a formalization of the same number by means of both $\underline{e}$ and ' $31,858,749,840,007,945,920,321$ ' is clearly adequate in the context described, contra Iacona.
} 
tions is consistent with the different views of the distinction between logical and non-logical expressions (cf. Iacona 2018: 127f.). Presumably he is thinking of the fact that the representation of the truth condition of a given sentence will turn out to be the same regardless of how we draw the distinction between logical and non-logical expressions. However, even if this is so, it might be argued that some ways of drawing the distinction are inconsistent with the idea that logical form, in the "logical" sense of logical form, is given by a representation of truth conditions. I'm thinking of the fact that some characterizations of logicality, e.g. as topic-neutrality, would appear to imply that some indexicals, such as demonstrative pronouns, are logical expressions that ought to be represented as fixed, non-schematic parts in logical form. Iacona's view, by contrast (always under a direct reference view of the referential contribution of indexicals), requires that indexicals be represented as schematic parts in logical form. Thus, for example, on his view one adequate representation of the argument

This is white / This is white

is $\mathrm{Fa} / \mathrm{Fa}$ (if the referent of 'this' is the same in the two occurrences), another is $\mathrm{Fa} / \mathrm{Fb}$ (when the referent is different). But the mentioned views of logicality would require that the argument be formalized by something like $F$ (this) / F(this), making appropriate postulations in the model theory so as to contemplate the different possibilities as to identity or difference of reference of the two occurrences of 'this'.

This issue is connected to the question of the indefiniteness of the notion of adequate formalization. One way in which the notion may be indefinite may have to do with the indefiniteness of the notion of a logical expression. On a number of views, including my own view, ${ }^{6}$ this notion is fundamentally vague, and 'this' and other demonstrative pronouns may well be examples of borderline cases. If this is so, it will be arbitrary to a great extent whether to represent 'this' by a fixed or a schematic part in logical form. One of Iacona's key claims, by contrast, is that in cases where different occurrences of the same indexical in the same sentence or argument have different referents

${ }^{6}$ See Gómez-Torrente 2002, and the more positive account in GómezTorrente (forthcoming). 
it is only adequate to represent those occurrences by different individual constants in a logical formalization, and hence in logical form. In my view, this makes his view again liable to a complaint of arbitrariness. It may be appropriate to say, following Iacona, that in some cases $a=b$ is an appropriate formalization of This is the same as this. But it might also be postulated, without violating any real constraint on logical form, that this=this is an appropriate formalization provided we understand the model theory of 'this' appropriately, as involving the possibility of different referents for different occurrences, even in the same sentence. (Such a postulate does not commit us to the claim that the sentence This is the same as this has the logical form of a classically valid schema such as $c=c$; we can just note in our model theory that this=this is not an instance of the schema $c=c$.)

Let me say by way of conclusion that, contrary to Iacona, I believe that there are some indications that an appropriate understanding of the "logical" notion of logical form will not match a characterization made in terms of the notion of truth conditions. Some of the indications have just been mentioned. Other more general indications have to do with general reasons why the distinction between logical and non-logical expressions doesn't seem to correspond to a semantic difference (not even in a broad sense of 'semantic'), and has rather to do with pragmatic characteristics of expressions. ${ }^{7}$ I think this pragmatic orientation can also be applied to provide an understanding or reconstruction of some of the pre-theoretic ideas about logical form that we mentioned in the course of our criticisms of Iacona's claims above, such as the claim that (21) and (22) differ in logical form, that $\underline{\underline{e}}$ is a counterexample to Euler's conjecture and $31,858,749,840,007,945,920,321$ is a counterexample to Euler's conjecture differ in logical form, and that Hesperus is a star and Phosphorus is a star differ in logical form. But this is of course not the place for me to argue for my own views on logical form; a development of these themes must be left for another occasion.

Mario Gómez-Torrente Instituto de Investigaciones Filosóficas National Autonomous University of Mexico (UNAM)

\footnotetext{
${ }^{7}$ See again Gómez-Torrente 2002 and Gómez-Torrente (forthcoming).
} 


\section{References}

Abbott, B. 2010. Reference. New York: Oxford University Press.

Gómez-Torrente, M. 2002. The problem of logical constants. Bulletin of Symbolic Logic 8: 1-37.

Gómez-Torrente, M. 2019. Roads to Reference. An Essay on Reference Fixing in Natural Language. Oxford: Oxford University Press.

Gómez-Torrente, M. Forthcoming. The problem of logical constants and the semantic tradition: from invariantist views to a pragmatic account. In The Semantic Conception of Logic, ed. by G. Sagi and J. Woods. Cambridge: Cambridge University Press, forthcoming.

Iacona, A. 2018. Logical Form. Between Logic and Natural Language, Cham: Springer.

Richard, M. 1986. Quotation, grammar, and opacity. Linguistics and Philosophy 9: 383-403.

Soames, S. 1987. Direct reference, propositional attitudes and semantic content. Philosophical Topics 15: 47-87. Reprinted in his Philosophical Papers, Vol. II. The Philosophical Significance of Language, Princeton (N.J.): Princeton University Press, 2009, 33-71. Reference to the reprint. 